[0212-7199 (2004) 21: 3; pp 118-122] ANALES DE MEDICINA INTERNA Copyright @ 2004 ARAN EDICIONES, S.L.

An. Med. InTERna (Madrid) Vol. 21, N. ${ }^{\circ}$ 3, pp. 118-122, 2004

\title{
Riesgo de infecciones y control metabólico en pacientes con diabetes mellitus tipo 2
}

\author{
M. C. MARTÍN MUÑOZ, A. GÓMEZ DE LA CÁMARA, A. ROMÁN MARTÍNEZ, \\ P. FERRANDO VIVAS, M. E. ALBARRÁN JUAN, F. HAWKINS \\ Centro de Salud Abrantes. Servicio de Endocrinología. Hospital Universitario 12 de \\ Octubre (Área 11). Madrid
}

\section{RESUMEN}

Fundamento y objetivo: La asociación entre diabetes mellitus (DM) tipo 2 y la prevalencia de infecciones es una situación frecuente a considerar en la practica clínica. Sin embargo, la evidencia de esta mayor susceptibilidad en los diabéticos para contraer infecciones es escasa. El objetivo de este estudio ha sido analizar el riesgo de infección en pacientes DM tipo 2 según el grado de control glucémico ( $\mathrm{Hb} \mathrm{Aic} \%$ ).

Pacientes y método: Estudio de cohortes históricas sobre 740 pacientes. Se evaluaron las tasas de incidencia de episodios infecciosos en diabéticos tipo 2 durante un periodo de 5 años, desde mayo de 1997 hasta mayo de 2002.

Resultados: La media de seguimiento en los diabéticos fue de 4,26 años. No se encontraron diferencias significativas en la presentación de infecciones en relación al control metabólico de los pacientes $(\mathrm{p}=0,33)$. Existe un mayor riesgo de infección en diabéticos con un número de procesos crónicos asociados elevado $(\mathrm{p}=0,035)$. Existe un mayor riesgo de infección cuanto más elevada es la edad del paciente $(\mathrm{p}=0,007)$ y a más años de evolución de la enfermedad $(\mathrm{p}=0,012)$. Se observó que los pacientes tratados con medidas higiénico-dietéticas sin otro tratamiento farmacológico tenían mejores controles de $\mathrm{Hb} \mathrm{Aic} \%(\mathrm{p}=0,0001)$, y menor riesgo de infecciones $(\mathrm{p}=0,038)$ que los tratados con insulina más ADO combinados.

Conclusiones: No existe correlación entre el grado de control glucémico $(\mathrm{Hb} \mathrm{Aic} \%)$ con la frecuencia de infecciones en los pacientes diabéticos estudiados. Sí aparece un mayor riesgo de infección en aquellos con un número de procesos crónicos asociados elevado.

PALABRAS CLAVE: Diabetes Mellitus tipo 2. Infección. Riesgo. Estudio de cohorte. Prevalencia.
RISK OF INFECTIONS AND GLYCEMIC CONTROL IN TYPE 2 DIABETES MELLITUS

ABSTRACT

Background and objective: Although association between Diabetes Mellitus (DM) type 2 and prevalence of infections is a frequently considered issue in current literature there is few evidence of it. The objective of this study has been to analyse the infection risk in DM type 2 patients according to their glycemic control level ( $\mathrm{Hb} \mathrm{Aic \% ).}$

Patients and method: In this cohort-study of 740 patients, we evaluated the incidence rates of infectious episodes in DM2-type diabetic patients during a period of 5 years, from May, 1997 to May, 2002.

Results: The average period of follow-up was 4.26 years. We found no significant correlation ( $p=0.33$ ) between higher levels of $\mathrm{HbAic} \%$ and presentation of infections. Data clearly show a direct correlation between risk of infection and number of associated chronic diseases $(p=0.035)$, age $(p=0.007)$ and diabetes duration (0.012). We also found an unexplained association between more aggressive treatment and higher glycemic levels ( $\mathrm{Hb} \mathrm{Aic \% )} \mathrm{(} p=0.0001)$ and also higher infection risk (0.038).

Conclusions: Although there is no association between risk of infection and glycemic control (Hb Aic\%) among analysed patients, this study shows a clear correlation between risk of infection and number of complications or number of associated chronic diseases.

KEY WORDS: Diabetes Mellitus type 2. Infection. Risk. Cohort-study. Prevalence.

Martín Muñoz MC, Gómez de la Cámara A, Román Martínez A, Ferrando Vivas P, Albarrán Juan ME, Hawkins F. Riesgo de infecciones y control metabólico en pacientes con diabetes mellitus tipo 2. An Med Interna (Madrid) 2004; 21: 118-122.

\section{INTRODUCCIÓN}

La asociación entre diabetes mellitus (DM) y el riesgo de infecciones es aceptada como frecuente en la práctica clínica habitual (1). Sin embargo la evidencia para soportar dicha asociación es escasa (2). Existen estudios que apoyan la idea de una mayor susceptibilidad y frecuencia para las infecciones bacterianas, mientras que otros hacen hincapié en la mayor severidad para las infecciones cuando éstas tienen lugar, en particular las provocadas por microorganismos no usuales incluyendo los hongos (3-5).

Un estudio reciente señala la existencia de un mayor riesgo de infecciones respiratorias y cutáneas cuando se compara con enfermos crónicos de otra patología (6).

Trabajo aceptado: 1 de octubre de 2003 